\title{
Analysis of Modified Bowtie Nanoantennas in the Excitation and Emission Regimes
}

\author{
Karlo Q. da Costa, Victor A. Dmitriev, \\ Federal University of Para,Belém-PA,Brazil,e-mails: karlo@ufpa.br,victor@ufpa.br
}

\begin{abstract}
In this work, we analyze modified bowtie nanoantennas with polynomial sides in the excitation and emission regimes. In the excitation regime, the antennas are illuminated by an incident plane wave, and in the emission regime, the excitation is fulfilled by infinitesimal electric dipole positioned in the gap of the nanoantennas. Several antennas with different sizes and polynomial order were numerically analyzed by method of moments. The results show that these novel antennas possess a controllable resonance by the polynomial order and good characteristics of near field enhancement and confinement for applications in enhancement of spontaneous emission of a single molecule.
\end{abstract}

Index Terms - Nanoantennas; modified bowtie nanodipoles; near field enhancement; spontaneous emission; method of moments.

\section{INTRODUCTION}

Nanoantennas are metal nanostructures used to enhance, confine, receive, and transmit optical fields [1]. Potential applications of nanoantennas are ultra-high-density optical data storage devices [2], super-resolution microscopy [3], integrated nano-optical devices [4], optical communication between functional elements of nanometer size [5], chemical enhancement for surface-enhanced Raman scattering [6], and biology [7]. Increasing investigations in this field in the last years is due to the promising applications, the development of modern techniques of micro and nanofabrication tools such as focused ion beam milling (FIB), and increasing the capabilities of numerical techniques of analysis.

One important application of nanoantennas is to enhance the spontaneous emission of a single molecule. In this process, a molecule positioned near a given antenna is excited by the high local field of the antenna. The analysis of this process can be divided in two regimes: excitation and emission ones. In the first regime, an incident plane wave illuminates the antenna and the near field enhancement is the phenomenon to be investigated. In the second regime, the emitter localized near the antenna is modeled by an infinitesimal electric dipole, and the radiation efficiency is the object of interest. The whole emission performance of the emitter depends on these two processes. Analysis of linear and bowtie nanoantennas for the enhancement of spontaneous emission are given in [8], [9].

In this paper, we present an analysis of Au modified bowtie nanoantennas in the excitation and emission regimes. The proposed antennas possess polynomial sides instead of linear of the conventional triangular antenna. We numerically study several modified antennas with different sizes 
and polynomial order by method of moments $(\mathrm{MoM})$. The analyzed parameters are: spectral response, resonances, near field enhancement and confinement, radiation efficiency and directivity.

\section{THEORY}

\section{A. Geometries of the Modified Bowtie Nanantennas}

The geometries of the modified bowtie nanoantennas are shown in Fig. 1. In this figure, four antennas made of $\mathrm{Au}$ with different values of the parameter $\alpha=(1,2,3,4)$ are presented, where $\alpha$ is the polynomial order of the sides variation. The polynomial function used to model the curvature of the sides is $x=\left(y / k_{1}\right)^{\alpha}$, where $k_{1}=L / 2 h^{1 / \alpha}, h=0.5 L(3)^{0.5}, L$ is the side length of the conventional bowtie triangle. The conventional bowtie antenna corresponds to the case $\alpha=1$ where the side variation is linear. These antennas are placed in the origin of the coordinate system with the axis of the antenna oriented along the $x$ axis. The distance between the arms of the bowtie dipoles is $d$, and the thickness, which is not shown, is $w$. With these parameters, the total antenna's length is $d+2 \times h$. For higher values of $\alpha$, the tips of the antenna are more acute.

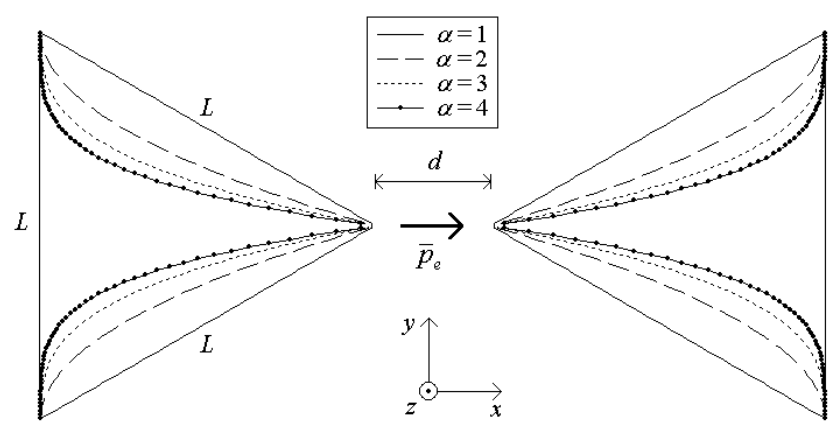

Fig. 1. Geometries of the bowtie nanoantennas with polynomial sides. The form of the sides varies as the function $x=\left(y / k_{1}\right)^{\alpha}$, where $k_{1}=L / 2 h^{1 / \alpha}, h=0.5 L(3)^{0.5}$. The conventional bowtie antenna corresponds to $\alpha=1$.

\section{B. Numerical Model of Analysis}

The nanoantennas shown in Fig. 1 were numerically analyzed by a MoM code based on model proposed in [10]. In this model, the equivalent polarization current inside the total volume $v$ of the antennas

$$
\bar{J}_{e q}=j \omega\left[\varepsilon(\bar{r})-\varepsilon_{0}\right] \bar{E}(\bar{r})=\tau(\bar{r}) \bar{E}(\bar{r})
$$

is determined by solving the tensor integral equation for the electric field

$$
\left[1+\frac{\tau(\bar{r})}{3 j \omega \varepsilon_{0}}\right] \bar{E}(\bar{r})-P V \int_{v} \tau\left(\bar{r}^{\prime}\right) \bar{E}(\bar{r}) \cdot \bar{G}\left(\bar{r}^{\prime}, \bar{r}^{\prime}\right) d v^{\prime}=\bar{E}^{i}(\bar{r})
$$

In (2), $P V$ means the principal value of the integral where the evaluation is inside the volume $v$ excluding the singularities of the free-space vector Green's function $\bar{G}$. The variables and parameters 
in (1)-(2) are as follows: $\bar{E}^{i}$ and $\bar{E}$ are the incident and total electric field inside the volume $v$, respectively, $\omega$ the angular frequency, $k_{0}=\omega\left(\mu_{0} \varepsilon_{0}\right)^{1 / 2}=2 \pi c / \lambda$ the wave number, $\lambda$ is the wavelength, $c$ is the speed of the light in free space, $\mu_{0}$ the free space permeability, $\varepsilon_{0}$ the free space permittivity. The complex permittivity of the Au particles is $\varepsilon=\varepsilon_{0} \varepsilon_{r}$, where $\varepsilon_{r}$ is defined

$$
\varepsilon_{r}=1-\frac{\omega_{p 1}^{2}}{\omega^{2}-j \Gamma \omega}+\frac{\omega_{p 2}^{2}}{\omega_{0}^{2}-\omega^{2}+j \gamma \omega}+\varepsilon_{\infty}
$$

and the parameters of this equation are: $\varepsilon_{\infty}=6, \omega_{p 1}=13.8 \times 1015 \mathrm{~s}^{-1}, \Gamma=1.075 \times 1014 \mathrm{~s}^{-1}, \omega_{0}=2 \pi c / \lambda_{0}$, $\lambda_{0}=450 \mathrm{~nm}, \omega_{p 2}=45 \times 1014 \mathrm{~s}^{-1}$, and $\gamma=9 \times 1014 \mathrm{~s}^{-1}$ [1]. This model is a good approximation in the range of wavelengths from $500 \mathrm{~nm}$ to $1000 \mathrm{~nm}$. Exactly in this frequency range we fulfill our analysis.

The excitation of the antennas is represented by $\bar{E}^{i}$. Two kinds of sources are considered in this model: an Ex-polarized, z-directed plane wave, and an infinitesimal electric dipole with moment positioned in middle of the gap's antenna and polarized in the $x$ axis (Fig. 1). The first numerical experiment is called the excitation regime and the second the emission regime.

To solve these scattering problems by MoM, the volume of a given antenna is divided in $N$ small cubic subvolumes, where the total electric field is approximately constant. With this approximation, the integral equation is transformed into a linear system with $N_{t}=3 \mathrm{~N}$ equations because there are three electric field components in each subvolume.

\section{Definitions of the Parameters Analyzed}

The solution of the equivalent linear system produces the total electric field $\bar{E}$ inside the volume $v$ of the antenna. With this result, the near and far field characteristic parameters of the antennas are calculated. In the excitation regime, we calculated the near electric field and scattering cross section respectively by

$$
\begin{gathered}
\bar{E}^{t}(\bar{r})=\bar{E}^{i}(\bar{r})+\int_{v} \tau\left(\bar{r}^{\prime}\right) \bar{E}\left(\bar{r}^{\prime}\right) \cdot \bar{G}\left(\bar{r}, \bar{r}^{\prime}\right) d v^{\prime} \\
S C S(\theta, \phi)=8 \pi \eta \frac{U(\theta, \phi)}{\left|\bar{E}^{i}\right|^{2}}
\end{gathered}
$$

where $\eta$ is the free space impedance and $U(\theta, \phi)$ is the radiation intensity of the scattered far field in a given direction $\theta$ and $\phi$. Other characteristics analyzed in this regime are the field enhancement and spatial confinement. The first one is defined by $\left(|E| /\left|E_{0}\right|\right)^{2}$, where $|E|$ is the total field near the antenna and $\left|E_{0}\right|$ is the amplitude of the incident plane wave. The second characteristic is a spatial distance between $3 \mathrm{~dB}$ points where $\left(|E| /\left|E_{0}\right|\right)^{2}=0.5$. This parameter is a spatial bandwidth (in nanometers) around the maximum field enhancement, where in the case of the nanoantennas, this maximum value is in the middle of the antenna's gap. In the present analysis, we calculated the spatial confinement in the axis $y$ and $z$ (Fig. 1) and we represent them by $\sigma_{y}$ and $\sigma_{z}$, respectively. 
In the emission regime, we evaluated the directivity and radiation efficiency respectively by [11]

$$
\begin{gathered}
D(\theta, \phi)=4 \pi U(\theta, \phi) / P_{r a d} \\
e(\%)=100 \times P_{r a d} /\left(P_{l o s s}+P_{r a d}\right)
\end{gathered}
$$

where $P_{\text {rad }}$ and $P_{\text {loss }}$ are the radiated and dissipated power of the antennas. In (6) the characteristic $U$ is the total radiation intensity due to the scattered field by the antenna and the radiated field by the infinitesimal electric dipole.

\section{NUMERICAL RESULTS}

Based on theory presented in the previous section, several codes in Fortran and Matlab were developed. The modified nanoantennas with $\alpha=(1,2,3,4)$ were simulated with $N_{t}=4608,5460,6516$, and 7896, respectively. With these discretizations we have a good convergence of the results. The dimensions used in these simulations are $L=50 \mathrm{~nm}, d=10 \mathrm{~nm}$, and thickness $w=9.5 ; 10 ; 10.3 ; 10.6(\mathrm{~nm})$ for the antennas with $\alpha=(1,2,3,4)$ respectively. We also simulated nanoantennas with $\alpha=3$ for different values of $L$ and $d$. In these simulations, eight nanoantennas were analyzed with $L=75 ; 100 ; 125 ; 150$ $(\mathrm{nm})$ for fixed $d=10 \mathrm{~nm}$, and $d=5 ; 15 ; 20 ; 25(\mathrm{~nm})$ for fixed $L=50 \mathrm{~nm}$. The following sections present the results.

\section{A. Spectral Response in the Excitation Regime}

Fig. 2 shows the spectral response of the normalized field $\left(|E| /\left|E_{0}\right|\right)$ in the middle of the antenna's gap (the origin of the coordinate system) and the scattering cross section in the $+z$ direction $\left(S C S_{z}\right)$ of the modified bowtie nanoantenna with $\alpha=(1,2,3,4)$. These characteristics were obtained by (4) and (5) in the excitation regime. We observe in these results two principal resonances in $\lambda_{a}$ and $\lambda_{b}\left(\lambda_{a}>\lambda_{b}\right)$, which are the same for the near field $\left(|E| /\left|E_{0}\right|\right)$ and far field $\left(S C S_{z}\right)$.

These resonances are shifted to the right for large values of $\alpha$, but the variation of $\lambda_{a}$ is higher than $\lambda_{b}$. We conclude that the resonance $\lambda_{a}$ is more sensitive with the geometric parameter $\alpha$ than the resonance $\lambda_{b}$, and this parameter can be used to tune the resonance of a particular antenna.

The values of $|E| /\left|E_{0}\right|$ and $S C S_{z}$ at the resonances $\lambda_{a}$ and $\lambda_{b}$ are different. In the near field region, the main resonance is $\lambda_{a}$, where it presents highest values of $|E| /\left|E_{0}\right|$. In the far field region, the maximum values of $S C S_{z}$ are obtained in $\lambda_{b}$, the exception is the case $\alpha=2$ (Fig. 2). Table I summarizes the values of some parameters of these antennas at the resonance $\lambda_{a}$. This table shows that the modified dipoles with $\alpha=(2,3,4)$ have higher field enhancement than the conventional one $(\alpha=1)$, and the highest value is 692 of the case $\alpha=2$. The scattering cross section of antennas with larger values of $\alpha$ are smaller, this is due the different physical areas of the antennas. The spatial confinement presented in this table will be discussed in the next section. 

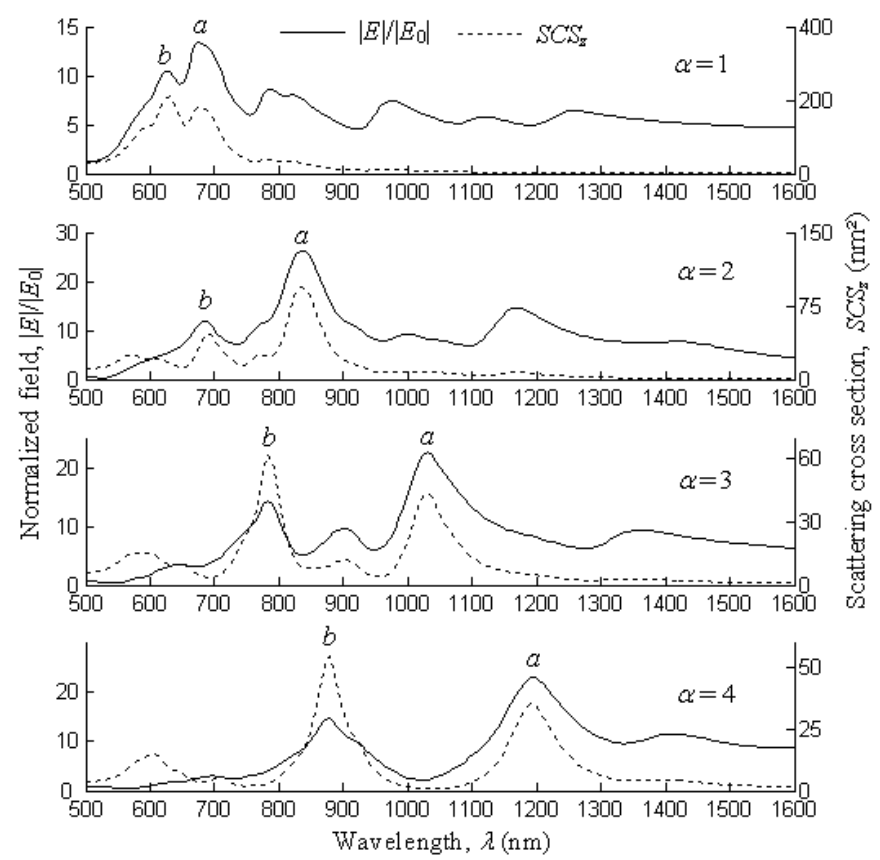

Fig. 2. Spectral response of the normalized field in the middle of the antenna's gap and scattering cross section in the direction $+z$ of the modified bowtie nanoantennas with $\alpha=(1,2,3,4)$ with $L=50 \mathrm{~nm}$ and $d=10 \mathrm{~nm}$.

TABLE I. PARAMETERS OF THE MODIFIED BOWTIE NANODIPOLES

\begin{tabular}{lllll}
\hline \multirow{2}{*}{ Parameter $^{\mathbf{a}}$} & \multicolumn{4}{c}{ Modified Bowtie Nanoantenna } \\
\cline { 2 - 5 } & $\boldsymbol{\alpha = \mathbf { 1 }}$ & $\boldsymbol{\alpha}=\mathbf{2}$ & $\boldsymbol{\alpha}=\mathbf{3}$ & $\boldsymbol{\alpha}=\mathbf{4}$ \\
\hline Resonant wavelength, $\lambda_{a}(\mathrm{~nm})$ & 675 & 840 & 1030 & 1194 \\
Field enhancement, $\left(|E| /\left|E_{0}\right|\right)^{2}$ & 180 & 692 & 508 & 524 \\
Scattering cross section, $S C S_{z}\left(\mathrm{~nm}^{2}\right)$ & 180 & 93 & 44 & 35 \\
Spatial confinement in $y, \sigma_{y}(\mathrm{~nm})$ & 7.7 & 5.8 & 6.9 & 7.6 \\
Spatial confinement in z, $\sigma_{z}(\mathrm{~nm})$ & 11 & 10.4 & 11 & 11.8 \\
\hline
\end{tabular}

a. These parameters were calculated at resonance $\lambda_{a}$.

Fig. 3 presents the dependence of the spectral response of the antenna with $\alpha=3$ on the lengths $L$ and $d$. The parameter shown in these figures is the normalized field $|E| /\left|E_{0}\right|$ in the middle of the antenna's gap. Fig. 4 shows the variation of the normalized field and resonant wavelength $\lambda_{a}$ versus the total antenna's length $2 \times h+d$ and the distance between the arms of the dipole $d$. We observe in these figures that when the size of the antenna is increased, the main resonance $\lambda_{a}$ and the normalized field are linearly increased. On the other hand, the variation of these parameters with $d$ are nearly constant for $\lambda_{a}$, and approximately exponential for $|E| /\left|E_{0}\right|$, where for lower distances $d$, this normalized field increases rapidly.

\section{B. Near Field Distributions}

In this section, we analyze the electric field distributions near the nanoantennas and its spatial confinement properties. Fig. 6 shows the variation of the normalized total field near the nanoantennas with $\alpha=(1,2,3,4)$ at the plane $z=10 \mathrm{~nm}$. This plane is approximately $5 \mathrm{~nm}$ above the antenna's surface, 
because the antenna's thickness are variable, i.e. $w=9.5 ; 10 ; 10.3 ; 10.6(\mathrm{~nm})$ for the antennas with $\alpha=(1,2,3,4)$ respectively. The dimensions of the antenna are $L=50 \mathrm{~nm}$ and $d=10 \mathrm{~nm}$, and the wavelength where these distributions were calculated are the resonances $\lambda_{a}$ given in Table I. In this figure, we observe the better field confinement of the modified antennas than that for the conventional one.

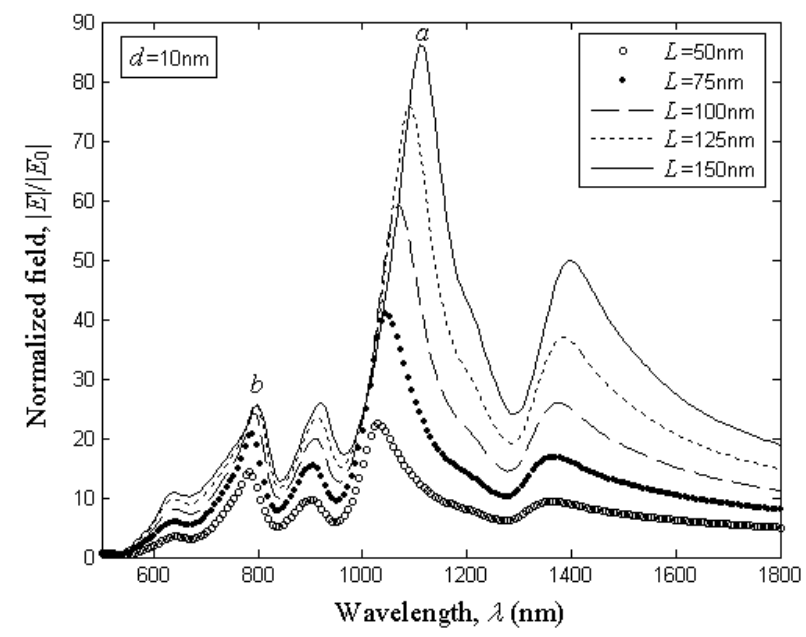

(a)

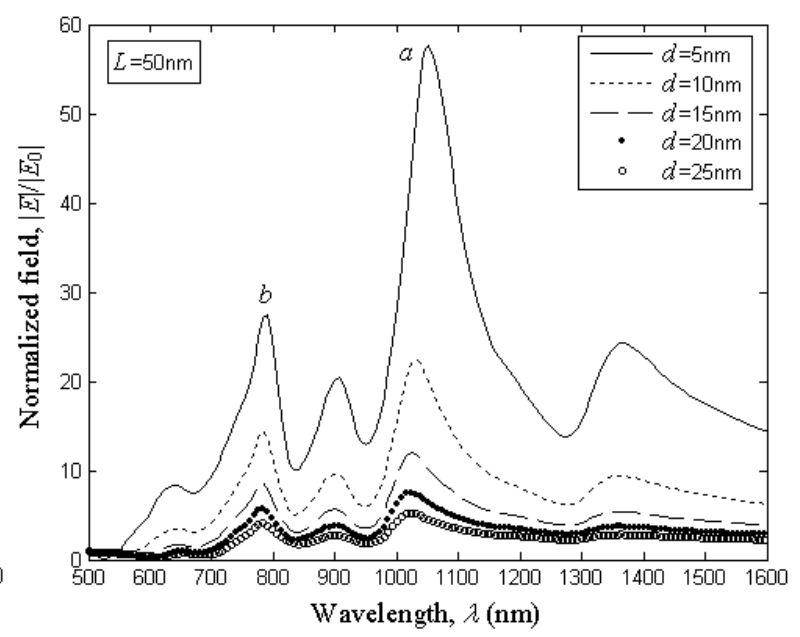

(b)

Fig. 3. Variation of the spectral response of the normalized field in the middle of the antenna's gap for the nanoantenna with $\alpha=3$. (a) variation with the length $L$. (b) variation with the length $d$.

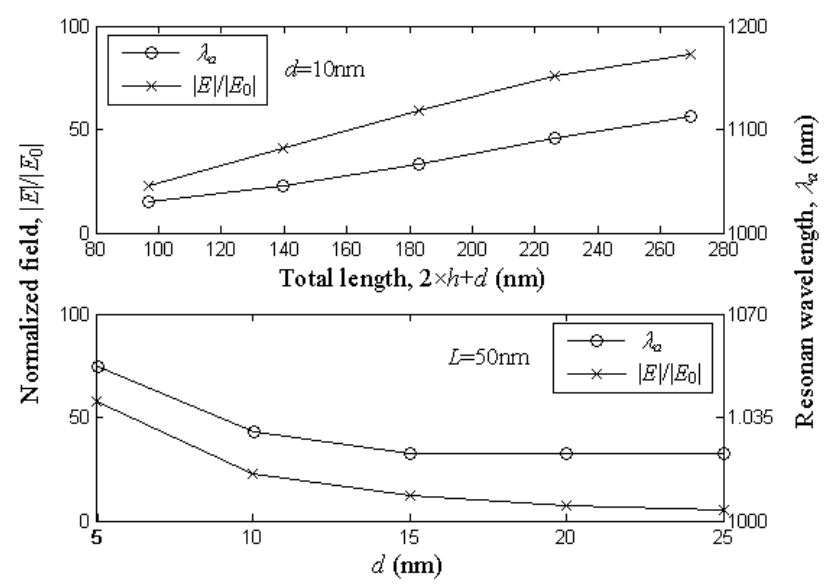

Fig. 4. Variation of the normalized field and resonant wavelength $\lambda_{a}$ versus the total antenna's length $2 \times h+d$ (upper) and distance between the arms of the nanoantenna $d$ (down). These results are for the antenna with $\alpha=3$.

The fields shown in Fig. 5 are the total field, but we also analyzed the distributions of the isolated components $x, y$, and $z$ for the antenna with $\alpha=3$. The results are presented in Fig. 6 , where we observe that the values of the components $x$ and $z$ are larger than the component $y$ in the plane $z=10 \mathrm{~nm}$ (Fig. 6(b)-(d)), and the $x$ component (Fig. 6(b)) is more confined in the antenna's gap. We also observe that there is only the component $x$ at the plane $y z$ between the arms of the dipole (Fig. 
6(a)). From these results, we conclude that in the antenna's gap, the principal polarization is the component $x$. If a molecule is positioned in the middle of this dipole, the antenna's field will excite in the molecule a electric dipole moment $\bar{p}_{x}$, this is why we analyze in this paper only this polarization. The results of this emission regime will be presented in the next section.

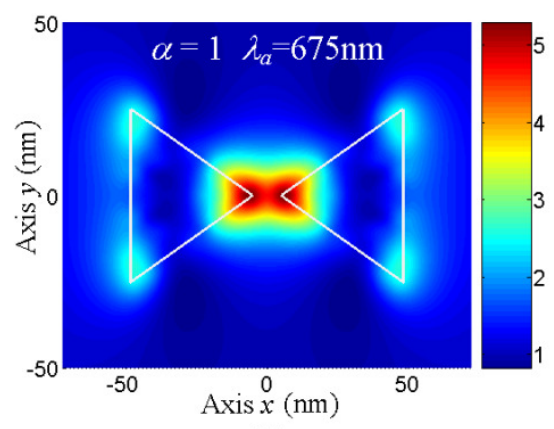

(a)

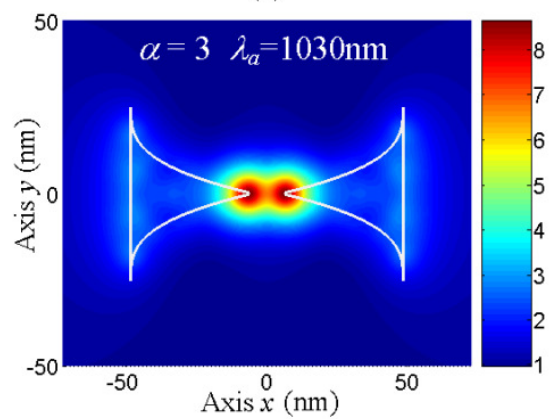

(c)

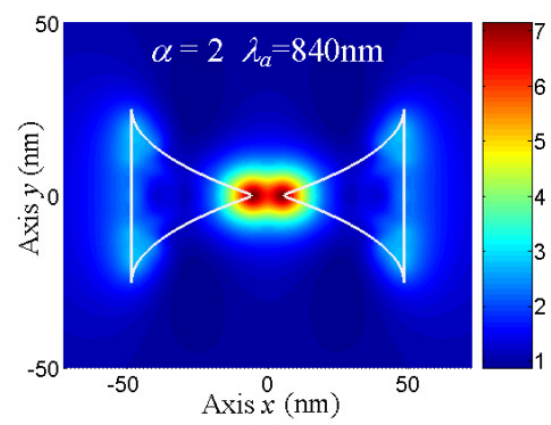

(b)

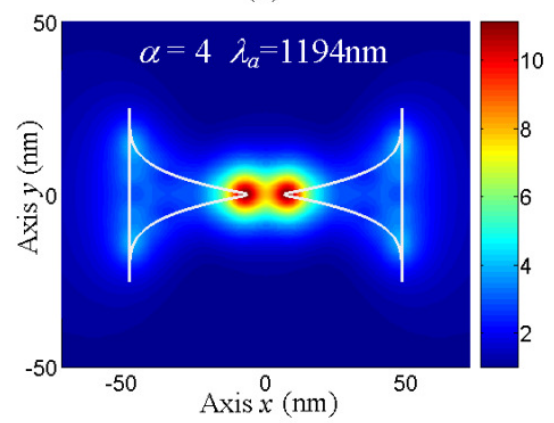

(d)

Fig. 5. Normalized near field $|E| /\left|E_{0}\right|$ distribution at the plane $z=10 \mathrm{~nm}$ of the modified nanoantennas with (a) $\alpha=1$, (b) $\alpha=2$, (c) $\alpha=3$, and (d) $\alpha=4, L=50 \mathrm{~nm}$ and $d=10 \mathrm{~nm}$.

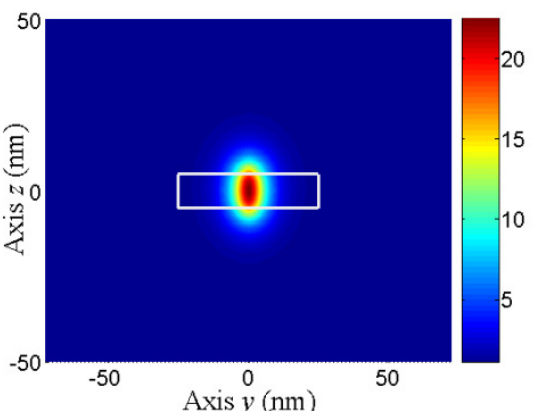

(a)

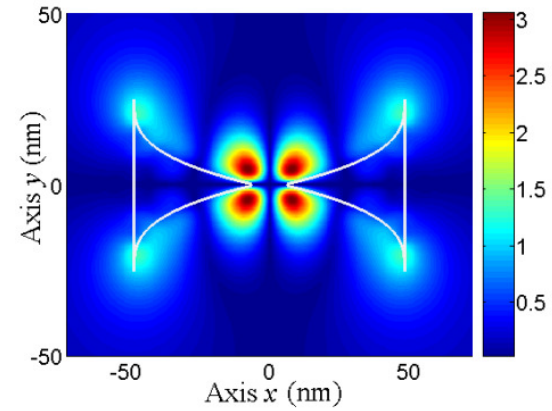

(c)

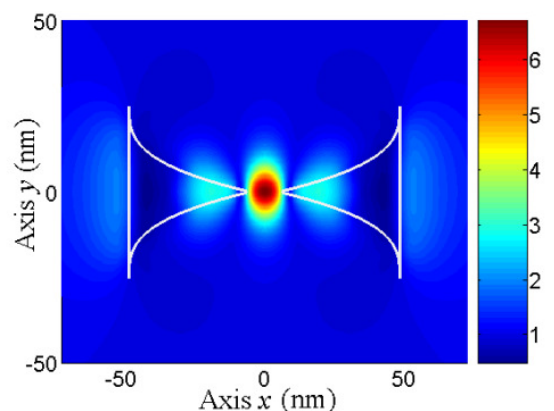

(b)

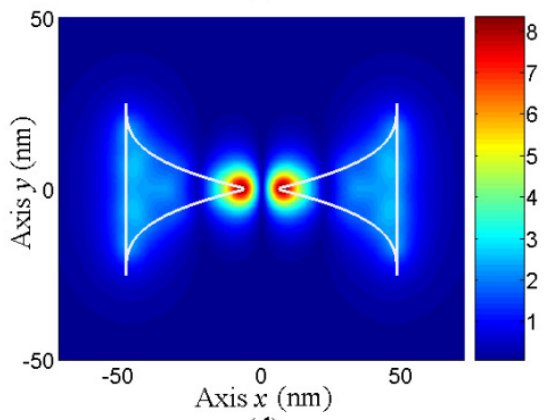

(d)

Fig. 6. Near field distributions of the nanoantenna with $\alpha=3$. (a) $\left|E_{x}\right| /\left|E_{0}\right|$ in $y z$, and (b) $\left|E_{x}\right| /\left|E_{0}\right|$, (c) $\left|E_{y}\right| /\left|E_{0}\right|$, (d) $\left|E_{z}\right| /\left|E_{0}\right|$ in $z=10 \mathrm{~nm}, L=50 \mathrm{~nm}$ and $d=10 \mathrm{~nm}$. 
To analyze the spatial confinement of the results presented in Fig. 5 and Fig. 5(a), we present in Fig. 7 the variation of the normalized field along the axis $y$ and $z$. The results of the spatial confinement parameters $\sigma_{y}$ and $\sigma_{z}$ are presented in Table I. We note that these antennas possess higher confinement along the axis $y$ than the axis $z$, and the highest confinement in the axis $y$ is for the modified antennas with $\alpha=2$, where $\sigma_{y}=5.8 \mathrm{~nm}$. The confinement in the axis $y$ depends on the value of the polynomial order $(\alpha)$, and the confinement in the axis $z$ depends on the antenna's thickness $(w)$. All the antennas in Fig. 6 possess approximately the same values of $w$, this is why they have similar values of $\sigma_{z}$.
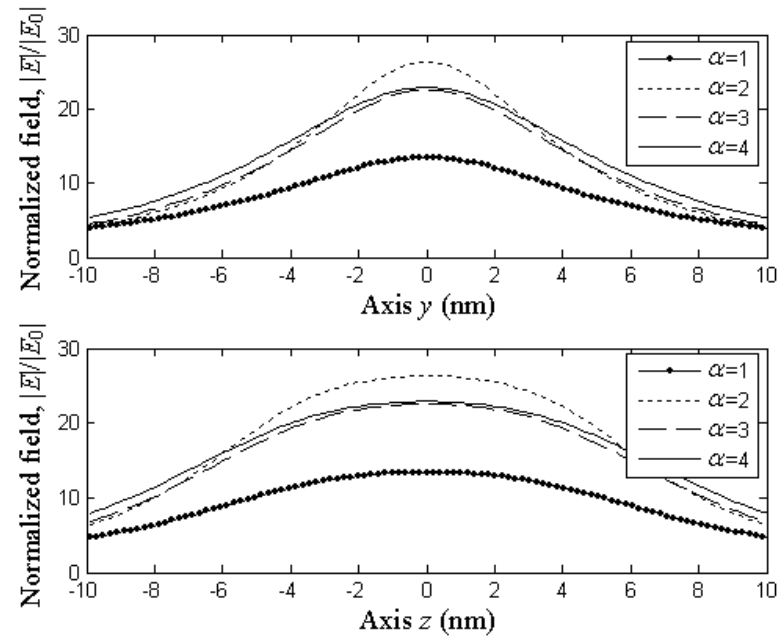

Fig. 7. Near field distributions $|E| /\left|E_{0}\right|$ of the nanoantennas with $\alpha=(1,2,3,4)$ along the axis $y$ (upper) and $z$ (down).

\section{Directivity and Efficiency in the Emission Regime}

In this section, we present the numerical results of the modified bowtie nanoantennas in the emission regime, where an infinitesimal electric dipole localized in the middle of the nanoantennas is the source of the antenna system (Fig. 1). The polarization of this infinitesimal dipole is in the $x$ direction and its moment dipole is $\bar{p}_{x}$. This polarization was chosen because in the excitation mode only the component $x$ of the electric field exists in the middle of the bowtie nanoantennas (Fig. 6). The results presented here are the directivity $(D)$ in the $+z$ direction $\left(\theta=0^{\circ}\right)$ and the radiation efficiency (e) as defined in (6) and (7) respectively.

Fig. 8 presents the spectral response of $D$ and e of the bowtie nanoantennas with $\alpha=(1,2,3,4)$, for $L=150 \mathrm{~nm}$ and $d=10 \mathrm{~nm}$. The results of $e$ show that the maximum efficiency of each antenna occurs at the same resonant wavelengths $\lambda_{a}$ and $\lambda_{b}$ of the excitation regime. We also observe that the efficiencies in these resonances are reduced for larger values of $\alpha$, i.e. antennas with same dimensions $L$ and $d$ but higher near field enhancements have smaller radiation efficiency. These results are in accordance to those previously observed for conventional bowtie nanoantennas with different radius of curvature of the bow angles [9]. 

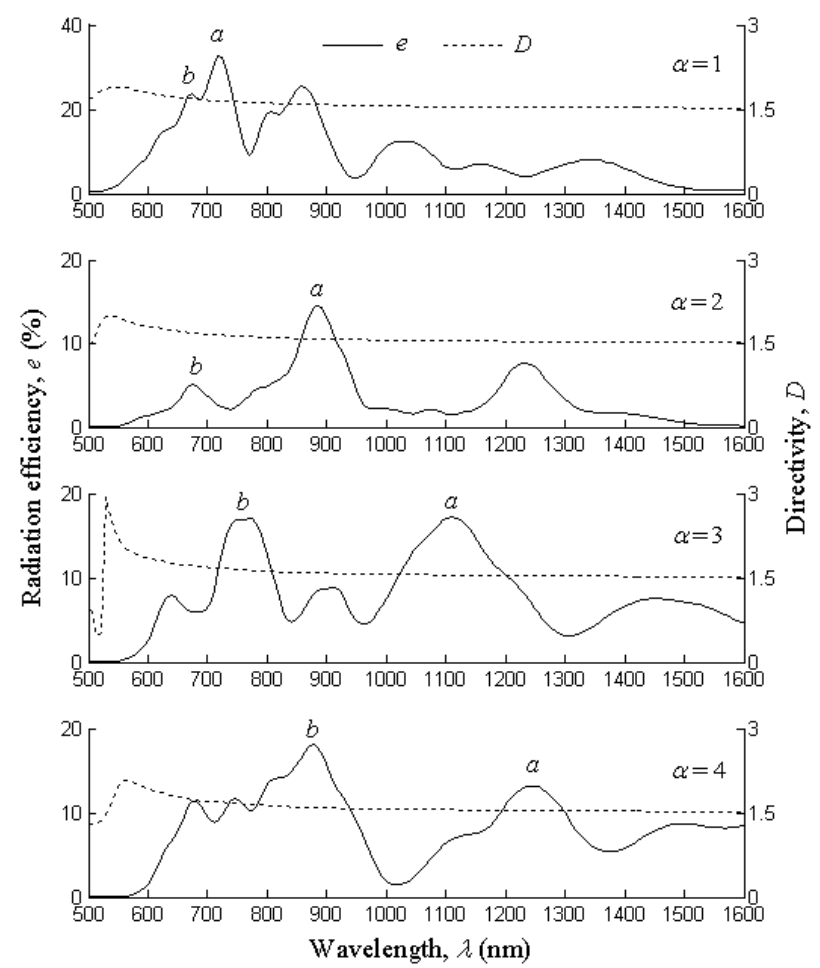

Fig. 8. Radiation efficiency and directivity in $+z$ direction versus wavelength of the nanoantennas with $\alpha=(1,2,3,4)$, for $L=150 \mathrm{~nm}$ and $d=10 \mathrm{~nm}$.

With respect the results of $D$ in Fig. 8 , all the antennas possess $D \approx 1.5$ at the resonances $\lambda_{a}$ and $\lambda_{b}$, this shows that these antennas have a radiation diagrams similar to that of an infinitesimal dipole in these resonances. The values of $D$ near the wavelength $\lambda=550 \mathrm{~nm}$ are a little different of 1.5 . We believe this is due the plasma resonance of $\mathrm{Au}$, but this effect is not important in the analysis because it occurs far from the resonance of the antennas, where the radiation efficiency is very small. In resume, the radiation characteristics of these antennas are similar to those of electrically small antennas, because the total length $2 \times h+d$ of them are smaller than the resonant wavelengths $\lambda_{a}$ and $\lambda_{b}$, so their directivities are constant in a wide range of wavelengths analyzed.

To investigate the dependence of $e$ on the dimensions $L$ and $d$, Fig. 9 show the variation of the spectral response of the radiation efficiency in function of the sizes $L$ and $d$. These results were obtained for the nanoantenna with $\alpha=3, d=10 \mathrm{~nm}$ in Fig. 9(a) and $L=50 \mathrm{~nm}$ in Fig. 9(b). We observe that the maximum efficiency also occur in the same resonant wavelengths $\left(\lambda_{a}\right.$ and $\left.\lambda_{b}\right)$ of the excitation regime presented in Fig. 4. For larger values of $L$ with $d$ fixed, we have better radiation efficiency, and for larger $d$ with $L$ fixed, we also have higher efficiency.

Fig. 10 presents the variation of $e$ at the resonances $\lambda_{a}$ and $\lambda_{b}$ in function of the total length $2 \times h+d$ and $d$. The results of these figures shows that the radiation efficiency is very small for nanoantennas with $L=50 \mathrm{~nm}$, for example in $d=10 \mathrm{~nm}$ the efficiency is $e<1 \%$, but it increases exponentially with $d$. This approximate exponential variation is for the resonance $\lambda_{a}$, and for the resonance $\lambda_{b}$ the efficiency stays practically constant. 


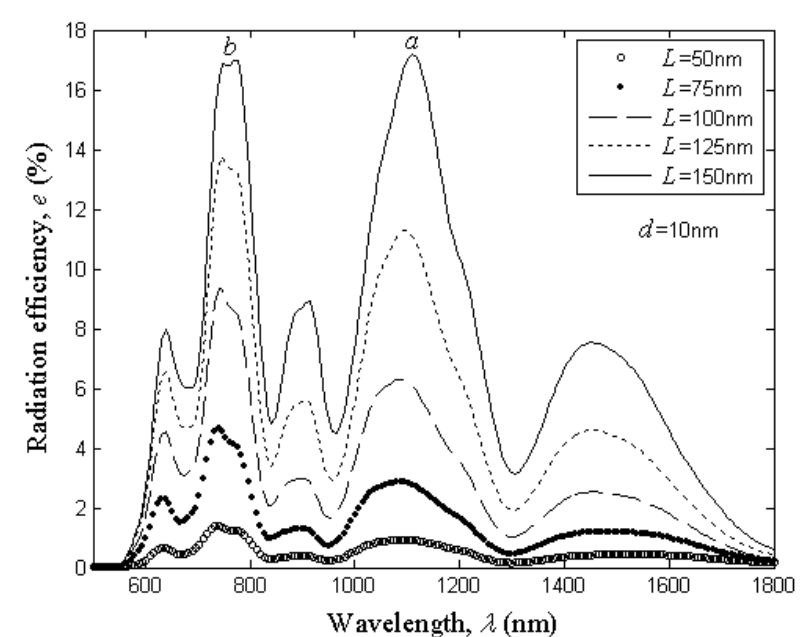

(a)

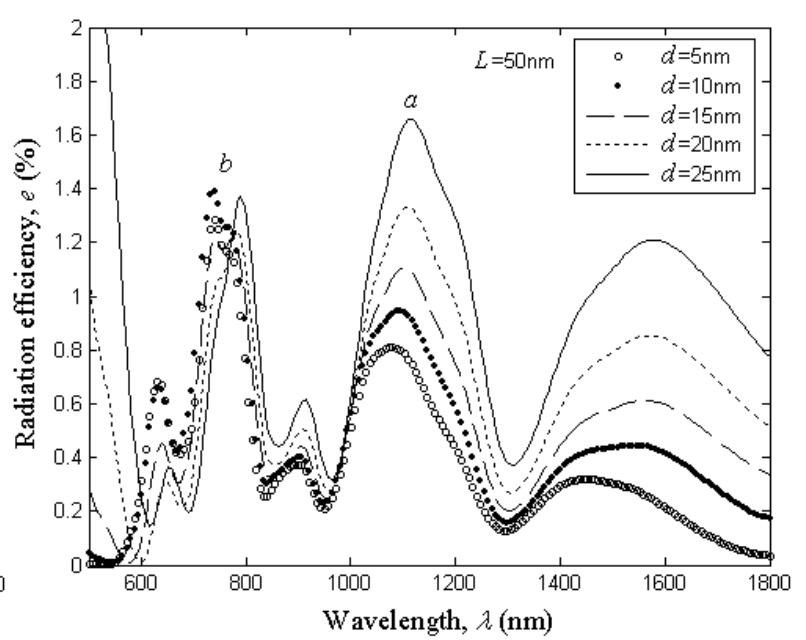

(b)

Fig. 9. Variation of the spectral response of the radiation efficiency of the nanoantenna with $\alpha=3$. (a) with the length $L$ for $d=10 \mathrm{~nm}$. (b) with the distance $d$ for $L=50 \mathrm{~nm}$.

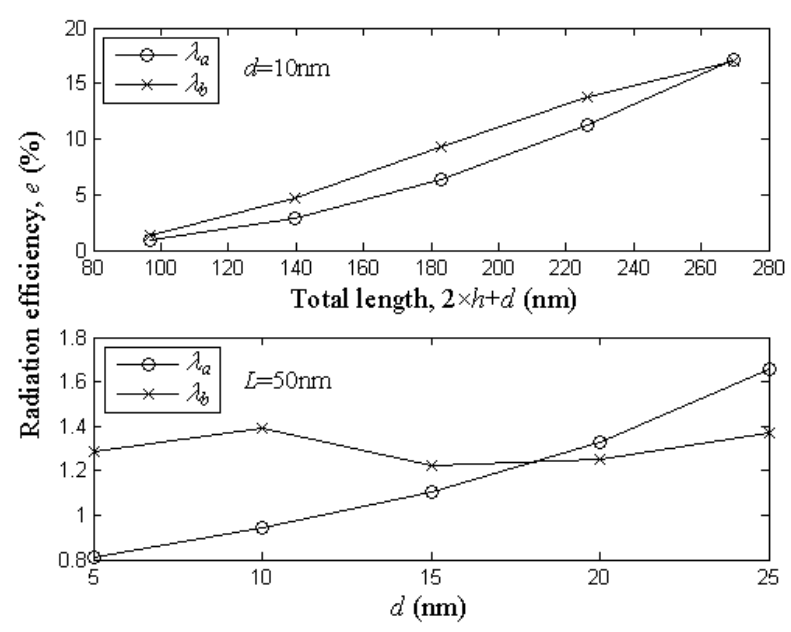

Fig. 10. Variation of the spectral response of the normalized field in the middle of the antenna's gap for the nanoantenna with $\alpha=3$. (a) variation with the length $L$. (b) variation with the length $d$.

The increasing of $e$ with $2 \times h+d$ is approximately linear for the two resonances $\lambda_{a}$ and $\lambda_{b}$, where if, for example, the total length is increased by a factor of two, the efficiency is also increased two times.

With the results presented in this section, we conclude that the modified bowtie nanoantennas with $\alpha>1$ possess smaller radiation efficiency than that of the conventional one with $\alpha=1$ and their directivities are equal to 1.5 at the principal resonances. However, we can rise up the radiation efficiency with the increasing of the dimensions $L$ and $d$.

\section{CONCLUSIONS}

In this paper, we presented a theoretical analysis of modified bowtie nanoantennas with polynomial sides in the excitation and emission regimes. We observed in the excitation regime that the resonances of the modified antennas are shifted to higher wavelengths and the electric field in the middle of the 
gap's antenna is increased, where it presents only polarization along the axis of the dipoles. Also, the field confinements of the modified antennas are higher than the conventional one. In the emission mode, we noted that the maximum radiation efficiency of all antennas occurs at the same resonant wavelengths obtained in the excitation regime, and the values of these efficiencies, for the modified antennas, are smaller than those of the conventional one with same dimensions, but it can be increased for larger antenna's size and gap distances between the arms of the dipoles. With respect the directive properties of these antennas, all the cases analyzed presented directivities similar to that of an electrically small antenna.

With all these results, we conclude that the modified bowtie nanoantennas have higher near field enhancement and confinement, and smaller radiation efficiency than those of the conventional bowtie antenna with linear sides. With appropriated dimensions, these novel nanoantennas can be used, for example, to enhance the spontaneous emission of single molecules positioned in the middle of the antenna's gap. Future works can be the analysis the characteristics dependence of these antennas on the thickness, orientation of the incident plane wave, and the polarization of the emitter in the gap.

\section{ACKNOWLEDGMENT}

This work was financially supported by the Brazilian agency National Counsel of Development Scientific and Technologic - CNPq by contract number 151731/2008-0.

\section{REFERENCES}

[1] L. Novotny, and B. Hecht, Principles of Nano-Optics, New York: Cambridge, 2006.

[2] H. Wang, C. T. Chong, and L. Shi, "Optical antennas and their potential applications to 10 terabit/in ${ }^{2}$ recording," Optical Data Storage Topical Meeting, pp. 16-18, May 2009.

[3] O. Sqalli, I. Utke, P. Hoffmann, and F. M.-Weible, "Gold elliptical nanoantennas as probes for near field optical microscopy," J. of Appl. Physics, vol. 92, pp. 1078-1083, July 2002.

[4] S. E. Lyshevski, and M. A. Lyshevski, "Nano- and microopto-electromechanical systems and nanoscale active optics," Third IEEE Conference on Nanotechnology, August 2003.

[5] J.-S. Huang, T. Feichtner, P. Biagioni, and B. Hecht, "Impedance matching and emission properties of nanoantennas in an optical nanocircuit," Nano Lett., vol. 9, pp. 1897-1902, April 2009.

[6] D. P. Fromm, A. Sundaramurthy, A. Kinkhabwala, P. J. Schck, G. S. Kino, and W. E. Moerner, "Exploring the chemical enhancement for surface-enhanced Raman scattering with Au bowtie nanoantennas," The J. of Chemical Phisics, vol. 124, 2006.

[7] M. F.G.-Parajo, "Optical antennas focus in on biology," Nat. Photonics, vol. 2, pp. 201-203, April 2008.

[8] J.-W. Liaw, “The quantum yield of a metallic nanoantenna,” Appl. Phys. A, vol. 89, pp. 357-362, 2007.

[9] J.-W. Liaw, "Analysis of a bowtie nanoantenna for the enhancement of spontaneous emission," IEEE J. Selected Top. Quantum Electronics, vol. 14, pp. 1441-1447, December 2008.

[10] D. E. Livesay, and K. M. Chen, "Electromagnetic fields induced inside arbitrary shaped biological bodies," IEEE Trans. Micro. Theo. Thec., vol. 22, pp. 1273-1280, December 1974.

[11] C. A. Balanis, Antenna Theory: Analysis and Design, 3rd ed., New Jersey: John Wiley, 2005. 